\title{
Exponential bulges and antitruncated disks in lenticular galaxies
}

\author{
Olga K. Sil'chenko ${ }^{1}$ \\ ${ }^{1}$ Sternberg Astronomical Institute, Moscow 119991, Russia \\ email: olga@sai.msu.su
}

\begin{abstract}
The presence of exponential bulges and anti-truncated disks has been noticed in many lenticular galaxies. In fact, it could be expected because the very formation of S0 galaxies includes various processes of secular evolution. We discuss how to distinguish between a pseudobulge and an anti-truncated disk, and also what particular mechanisms may be responsible for the formation of anti-truncated disks. Some clear examples of lenticular galaxies with a multi-tier exponential stellar structure are presented, - among them two central group giant S0s seen face-on and perfectly axisymmetric.
\end{abstract}

Keywords. Galaxies: elliptical and lenticular, cD; galaxies: bulges; galaxies: disks; galaxies: evolution

\section{Introduction}

Bulges have been traditionally thought to have de Vaucouleurs' brightness profiles just as elliptical galaxies (e.g. Freeman 1970). However John Kormendy (Kormendy 1982a, Kormendy 1982b, Kormendy 1993) has proved that there exists a type of bulges named 'pseudobulges' which resemble disks from the dynamical point of view: they are rather cold and demonstrate fast rotation. Despite their dynamical properties, they are bulges from the geometrical point of view: they are 'fat' and have rather large scaleheights. Pseudobulges are thought to be products of secular evolution; but if it is so, then dynamical simulations (e.g. Pfenniger \& Friedli 1991) predict that they must have exponential brightness profiles. And indeed, small bulges of late-type spirals which could be easily made by secular evolution, practically all have exponential brightness profiles (e.g. Andredakis et al. 1995, Graham 2001).

Not only small bulges in late-type spirals have exponential brightness profiles. Lenticular galaxies whose bulges usually are not small at all typically have mean Sersic coefficients lower than Sa galaxies, often with $n<2$ : the Sersic parameter $n$ peaks at the Sa-Sb morphological type and falls further toward S0s (Graham 2001, Laurikainen et al. 2005, Laurikainen et al. 2007). In fact, it is quite natural because the event of a galaxy transformation to S0 from a spiral must include various processes of secular evolution, resulting in radial matter re-distribution and reshaping of the bulge.

But galactic disks outside the bulges can also consist of several exponential segments. Now it becomes clear that so called anti-truncated disks, consisting of two exponential segments with the outer scalelength larger than the inner one, may be the dominant type of galactic disks among some types of galaxies (Erwin et al. 2008a). We reported finding a few large nearby spiral galaxies with such two-tiers disks during the last 10 years. A significant population of such disks has been found where the brightness profiles reach 27th or 28th magnitudes per square arcsecond (Pohlen \& Trujillo 2006). Does 
it mean that the outer segments of anti-truncated disks represent always low surfacebrightness (LSB) disks? The statistics by Erwin et al. (Erwin et al. 2008b) for barred galaxies says so. Among unbarred spiral galaxies, we find only one galaxy, NGC 5533, that has a LSB outer disk (Sil'chenko et al. 1998). Other galaxies (NGC 7217, Sil'chenko \& Afanasiev 2000; NGC 615, Sil'chenko et al. 2001; NGC 4138, Afanasiev \& Sil'chenko 2002; NGC 7742, Sil'chenko \& Moiseev 2006) have on the contrary quite normal outer disks as compared with the reference value of the central $B$ surface brightness of 21.7 (Freeman 1970); and simultaneously they have compact bright inner disks.

Can we distinguish the compact inner disk from a pseudobulge? Yes, by using a multivariant approach. For example, in NGC 7742 all spirals and current star formation are confined to the inner exponential disk, and the outer one is smooth and featureless (Sil'chenko \& Moiseev 2006), so we can conclude that the inner exponential stellar component is dynamically cold and cannot be a bulge. But the key property is a visible geometry of the stellar component with the exponential profile. To be a disk, it must be thin. For disks inclined to the line of sight, a good check is isophotal analysis. The outer disk is always assumed to be thin: then its isophote axis ratio characterizes the cosine of the inclination. If the inner component has the same visible axis ratio as the outer one and if two disks are coplanar, the inner structure cannot be a spheroid, it must be a disk. For face-on galaxies, this approach does not work: their isophotes are always round independent of the scaleheights. But for face-on galaxies we can use kinematical data and estimate their thickness by measuring vertical stellar velocity dispersions.

We have now started a program of studying systematically multi-tier (anti-truncated) exponential structures in early-type, presumably lenticular galaxies. The study will include photometric as well as spectral observations. Some first results are presented in this talk.

\section{Observations}

The photometric observations which we discuss here have been made with the focal reducer SCORPIO of the Russian $6 \mathrm{~m}$ telescope (Afanasiev \& Moiseev 2005) in the directimage mode. An EEV 42-40 CCD detector with $2048 \times 2048$ pixels was used, binning $2 \times 2$ pixels. The field of view was about 6 arcminutes, the scale 0.35 arcsec per binned pixel. The photometric observations were undertaken on August 21, 2007, with a seeing of about $2^{\prime \prime}$. We have exposed 5 fields in the NGC 80 group and 6 fields in the NGC 524 group, in the $B$ and $V$ filters. The exposure times were selected in accordance with the surface brightness of the targets observed; for example, we exposed NGC 524 itself during $60 \mathrm{sec}$ in the $B$-filter and during $30 \mathrm{sec}$ in the $V$-filter. As a flat field, we used the exposures of the twilight sky. The calibration onto the standard Johnson $B V$ system has been made by using multi-aperture photoelectric data collected by HYPERLEDA for NGC 80 and NGC 524 .

\section{Photometric structure of the central group S0 galaxies}

The two central group galaxies under consideration are typical giant lenticular galaxies, with blue absolute magnitudes of $\sim-21.6$ (HYPERLEDA). Both are very red, $(B-V)_{e}=$ 1.07 , and are seen face-on, $b / a>0.9$.

We have calculated azimuthally averaged surface brightness profiles for NGC 524 in two filters, $B$ and $V$. The data are rather precise, and we trace the profiles up to $R=80^{\prime \prime}$, or about $10 \mathrm{kpc}$ from the center, with an accuracy better than $0.01 \mathrm{mag}$. At larger radii 


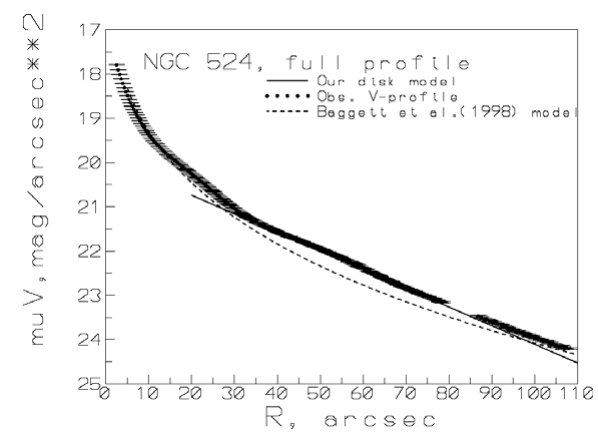

Figure 1. The V-band azimuthally-averaged brightness profile of NGC 524 obtained by us with the reducer SCORPIO at the $6 \mathrm{~m}$ telescope; the model decomposition proposed by Baggett et al. (1998) is overlaid. One can see that the high-accuracy data do not agree with a single de Vaucouleurs' bulge model; instead at least two exponential components are needed.
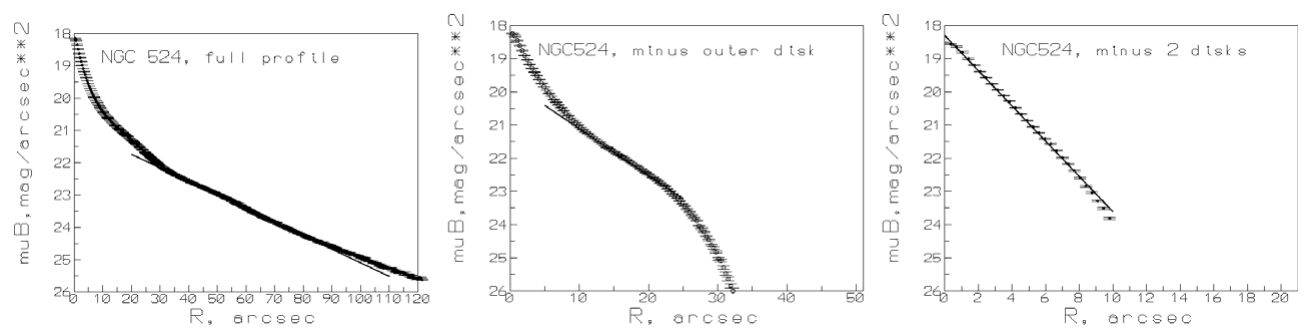

Figure 2. The B-band azimuthally-averaged brightness profile of NGC 524 obtained by us with the reducer SCORPIO at the $6 \mathrm{~m}$ telescope can be decomposed step-by-step into three exponential components.

the accuracy is worse due to bright stars projected onto the galaxy. Comparing our $V$ profile with the model decomposition proposed by Baggett et al. (Baggett et al. 1998) (Fig. 1), i.e. with a single de Vaucouleurs' bulge approximation, shows that their model is inappropriate. Instead we see at least two exponential components: the 'outer' one in the radius range $R=35^{\prime \prime}-90^{\prime \prime}$ and the 'inner' one in the radius range $R=10^{\prime \prime}-25^{\prime \prime}$.

We fit the outer part of the surface brightness profile by an exponential law, construct a 2D model image of this disk and subtract it from the observed full image. For the residual image, we calculate again the azimuthally averaged surface brightness profile, fit its outer part by an exponential law, construct the 2D image of the inner disk and subtract it from the first-step residual image. Interestingly, the brightness profile of the second-step residual image which is safely traced up to $10^{\prime \prime}$, is also exponential (Fig. 2)! Figure 2 presents all steps of our decomposition procedure, and in Table 1 we give the parameters of the exponential stellar substructures obtained by this procedure.

Table 1. Parameters of the photometric components of NGC 524 approximated by an exponential law

\begin{tabular}{lcccc}
\hline Component & Radius range of the fit & $\mu_{0}, \mathrm{mag} / \square^{\prime \prime}$ & $h^{\prime \prime}$ & $h, \mathrm{kpc}$ \\
\hline & & $B$-filter \\
Outer disk & $34^{\prime \prime}-90^{\prime \prime}$ & $20.906 \pm 0.007$ & $26.07 \pm 0.06$ & 3.02 \\
Inner disk & $10^{\prime \prime}-23^{\prime \prime}$ & $19.67 \pm 0.01$ & $7.67 \pm 0.05$ & 0.89 \\
Bulge & $1^{\prime \prime}-8^{\prime \prime}$ & $18.25 \pm 0.01$ & $2.04 \pm 0.01$ & 0.24 \\
\hline
\end{tabular}




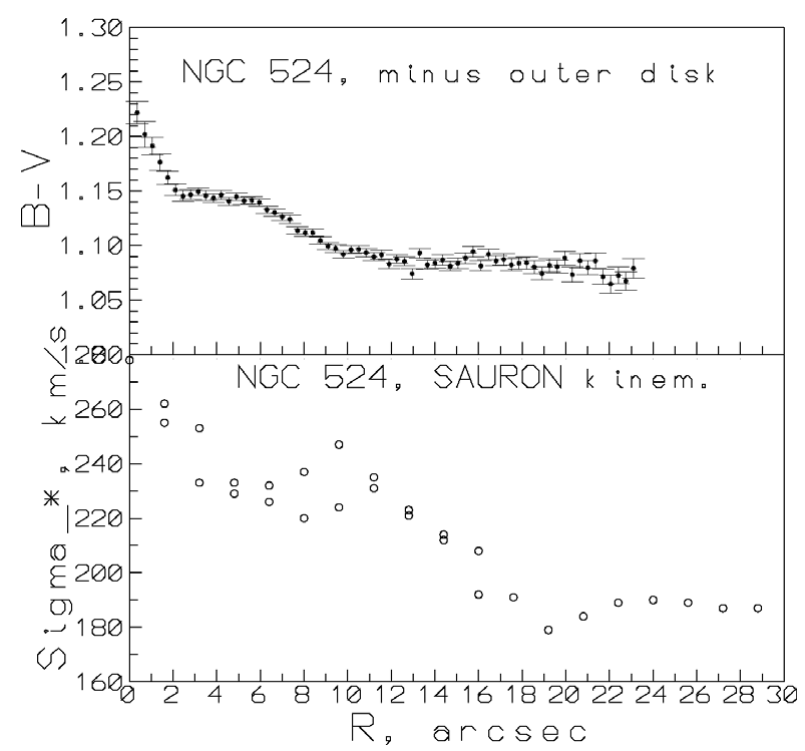

Figure 3. Comparison of the azimuthally averaged profiles in the $B-V$ colour, calculated from the residual image of NGC 524 after subtraction of the outer disk (upper), and the stellar velocity dispersion (lower), showing a break at the edge of a dynamically hot bulge.

Figure 3 presents the $B-V$ colour profile of the first-step residual image (the inner disk). It reveals a net colour difference between the inner disk, $R>10^{\prime \prime}$, and the 'bulge', $R<10^{\prime \prime}$. This colour difference, $\Delta(B-V)=0.07$, may result from a metallicity difference of 0.2 dex at an old stellar age $(T=12 \mathrm{Gyr})$, or alternatively from an age difference of 8 Gyr at a metallicity of $[\mathrm{Z} / \mathrm{H}]=+0.1$ (Worthey 1994, and also his WEBsite, Dial-a-Galaxy option), the inner disk being younger or/and more metal-poor.

We have compared the colour profile of the residual image with the stellar velocity dispersion profile similarly averaged in the rings over the map obtained from SAURON data (Emsellem et al. 2004, see also the WEBsite of the SAURON project) (Fig. 3). The profiles of the colour and of the stellar velocity dispersion are qualitatively similar! Certainly, we see a transition from the (exponential) bulge to the inner disk at $R \approx 10^{\prime \prime}$. Preliminary estimates of the scaleheight of the inner disk in NGC 524, by treating the measured line-of-sight stellar velocity dispersion as a vertical one, give a value of about $1.5 \mathrm{kpc}$; a typical value for a thick stellar disk as expected in a lenticular galaxy.

A very similar surface brightness profile, consisting of two exponential disks and a very compact bulge, has been observed by us earlier in the giant lenticular galaxy NGC 80, which is also located at the center of a rich X-ray group (Sil'chenko et al. 2003). Our new observations certainly confirm this multi-tier structure. In both galaxies the outer stellar disks are quite normal as regards their scalelengths or their central surface brightnesses, and the inner disks are compact and bright. Both galaxies are seen face-on and are certainly unbarred; the low ellipticity of their isophotes over the full radial extension proves that the galaxies are strictly axisymmetric. If the exponential inner stellar structures have been formed by secular evolution, where are the signatures of the main 'driver' of secular evolution, of a bar? 


\section{What can be the mechanisms for forming anti-truncated disks in lenticular galaxies?}

It seems clear that anti-truncated disks are the result of radial matter re-distribution in a disk galaxy, and the very event of re-distribution must be rather fast and discrete. Several candidate mechanisms can be proposed. Younger et al. (Younger et al. 2007) simulate a minor merger and obtain an anti-truncated stellar disk in the merger remnant, mainly due to stellar diffusion from the inner part of the initial spiral galaxy into an outer region. In such models the outer part of the multi-tier stellar disk in the merger remnant seems to be a low surface brightness disk.

Some years ago I proposed another mechanism where a transient interaction, due to, say, a passage of a rather massive galaxy, provokes intense gas inflow and results in the gas concentrating in the very inner part of a galaxy with a subsequent star formation burst. In this model, the inner disk must be brighter and more compact than the usual large-scale stellar disks of spiral galaxies. We may expect that a statistical study of the parameters of inner and outer exponential disks in the anti-truncated galaxies would help to select a model.

In the group NGC 80, besides the central galaxy, we have analysed brightness profiles in more 10 lenticular galaxies, with absolute blue magnitudes from -17 to -20 . Among those, 7 S0s appear to possess two-tier exponential disks. Of these, three have compact inner disks and normal outer disks, while four have normal inner disks and LSB outer disks. Together with NGC 80 itself and with NGC 524, with their compact bright inner disks and extended normal ones, we obtain half-to-half preliminary statistics, which implies that the origin of the multi-tier exponential disks may be different in different galaxies.

\section{Conclusions}

If we assume that multi-tier exponential profiles are formed by secular evolution of galactic disks, the best place to search for them would be lenticular galaxies. Lenticular galaxies have had to reform their stellar disks during their secular transformation from $\mathrm{S}$ to S0; hence S0s must be the hosts of both multi-tier disks and pseudobulges. To select a particular mechanism for forming anti-truncated disks, we must have a better knowledge of the statistics of their properties, which still does not cover representative samples. Among the possible alternatives to the origin of multi-tier exponential profiles are minor mergers or tides (but the same alternative exists for the origin of S0s outside clusters!). To choose, we need to know the ratio between compact+normal and normal+LSB stellar disk combinations.

Secular redistribution of stars and other matter in disks is thought to be caused by bars: bars are usually generated by any interaction and even without interactions - by intrinsic instabilities. But almost all our galaxies with multi-tier disks are unbarred; the face-on S0s NGC 524 and NGC 80 are perfectly axisymmetric. And the samples of Erwin et al. (Erwin et al. 2008a, Erwin et al. 2008b) imply the same conclusion: among barred galaxies, the anti-truncated disks contribute one third of all, among unbarred - more than $50 \%$. This inconsistency is a complete puzzle yet.

\section{Acknowledgements}

The $6 \mathrm{~m}$ telescope is operated under the financial support of Science and Education Ministry of Russia (registration number 01-43). During our data analysis we used the Lyon-Meudon Extragalactic Database (HYPERLEDA) supplied by the LEDA team at the CRAL-Observatoire de Lyon (France), and the NASA/IPAC Extragalactic Database 
(NED) operated by the Jet Propulsion Laboratory, California Institute of Technology under contract with the National Aeronautics and Space Administration. The study of multi-tier galactic disks is supported by the grant of the Russian Foundation for Basic Research (RFBR), no. 07-02-00229a. My attendance at IAU Symposium no. 254 was made possible by an IAU grant.

\section{References}

Afanasiev, V. L. \& Sil'chenko, O. K. 2002, AJ 124, 706

Afanasiev, V. L. \& Moiseev, A. V. 2005, Astronomy Letters 31, 194

Andredakis, Y. C., Peletier, R. F., \& Balcells, M. 1995, MNRAS 275, 874

Baggett, W. E., Baggett, S. M., \& Anderson, K. S. J. 1998, AJ 116, 1626

Emsellem, E., Cappellari, M., Peletier, R. F., et al. 2004, MNRAS 352, 721

Erwin, P., Pohlen, M., Beckman, J. E., Gutiérrez, L., \& Aladro, R. 2008, in: J. H. Knapen, T.J. Mahoney, \& A. Vazdekis (eds.), Pathways through an Eclectic Universe. ASP Conf. Ser. v. 390 (San Francisco), p. 251

Erwin, P., Pohlen, M., \& Beckman, J. E. 2008, AJ 135, 20

Freeman, K. C. 1970, ApJ 160, 811

Graham, A. W. 2001, MNRAS 326, 543

Kormendy, J. 1982, in: L. Martinet \& M. Mayor (eds.), Morphology and Dynamics of Galaxies, Twelfth Advanced Course of the Swiss Society of Astronomy and Astrophysics (Sauverny: Geneva Obs.), p. 113

Kormendy, J. 1982, ApJ 257, 75

Kormendy, J. 1993, in: H. Habing \& H. Dejonghe (eds.), Galactic Bulges. IAU Symp. 153 (Dordrecht: Kluwer), p. 209

Laurikainen, E., Salo, H., \& Buta, R. 2005, MNRAS 362, 1319

Laurikainen, E., Salo, H., Buta, R., \& Knapen, J. H. 2007, MNRAS 381, 401

Pfenniger, D. \& Friedli, D. 1991, $A \&$ A 252, 75

Pohlen, M. \& Trujillo, I. 2006, A\& A 454, 759

Sil'chenko, O. K., Burenkov, A. N., \& Vlasyuk, V. V. 1998, New Astronomy 3, 15

Sil'chenko, O. K. \& Afanasiev, V. L. 2000, A\& A 364, 479

Sil'chenko, O. K., Vlasyuk, V. V., \& Alvarado, F. 2001, AJ 121, 2499

Sil'chenko, O. K., Koposov, S. E., Vlasyuk, V. V., \& Spiridonova, O. I. 2003, Astronomy Reports 47,88

Sil'chenko, O. K. \& Moiseev, A. V. 2006, AJ 131, 1336

Worthey, G. 1994, ApJS 95, 107

Younger, J. D., Cox, T. J., Seth, A. C., \& Hernquist, L. 2007, ApJ 670, 269 